\title{
Kinderkrippe schützt? Stimmt gar nicht!
}

\author{
Nach der Hygienehypothese haben Kinderkrippen und Kindergärten \\ einen protektiven Einfluss auf die Allergieentwicklung. Vermutet wird \\ unter anderem eine Stärkung des Immunsystems durch die dort \\ häufigeren Infektionen. In Schweden scheint das nicht zu gelten.
}

m Jahr 2000 wurde ein Fragebogen an Eltern von 14.077 schwedischen Kindern im Alter von ein bis sechs Jahren versandt. Ermittelt werden sollten Symptome von Atemwegs- und Allergieerkrankungen, Faktoren des häuslichen Umfelds und die Art der Kinderbetreuung. Die Auswertung der zurückgesandten 10.851 Fragebögen zeigte signifikant höhere Risiken für die 87,2\% der Kinder, die - fast zur Hälfte ganztags - in Krippen und Kindergärten betreut wurden: Die Kinder hatten in den letzten 12 Monaten häufiger Wheezing-Attacken (adjustierte Odds Ratio [AOR] 1,33), nächtlichen Husten, ohne erkältet zu sein (AOR 1,56) und eine ärztliche Asthma-, Rhinitis-, Heuschnupfen-, oder Ekzemdiagnose (AOR 1,23/1,15/1,75/ $1,49)$. Ebenfalls signifikant häufiger wurde über Nahrungsmittelallergien, mehr als sechs Erkältungen und Ohrinfektionen berichtet (AOR 1,27/2,57/2,14). Bei den Kinder bis vier Jahren waren die Unterschiede zwischen in der Krippe und zu Hause Betreuten besonders groß.

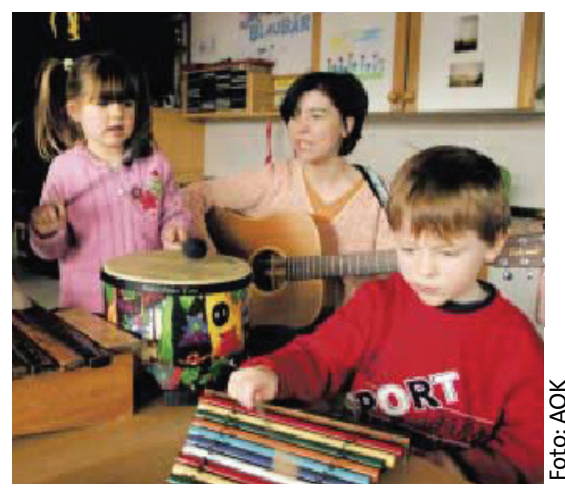

Kinderbetreuung in Gruppen hat Vorteile, eine Allergieprotektion scheint aber nicht dazu zu gehören.

\section{Probiotika: Immunstimulation belegt}

\section{Eine bakterielle Fehlbesiedlung des Darmes wird als eine Ursache für atopische Hauterkrankungen diskutiert. Probiotika können Neurodermitissymptome bessern helfen. Sie modulieren offensicht- lich die fehlgeleitete Immunreaktion im Darm.}

n $\mathrm{n}$ einer klinischen Studie wurden gezielt immunologische Effekte von Probiotika bei 230 Kindern mit einem atopischen Ekzem bzw. einer Kuhmilchallergie untersucht. Dazu erhielten die Kinder randomisiert über vier Wochen entweder Lactobacillus GG (LGG) oder eine Mischung aus vier verschiedenen Probiotika-Stämmen (LGG, Lactobacillus rhamnosus LC705, Bifidobacterium breve $\mathrm{Bb} 99$, Propionibacterium freudenreichii ssp. shermanii JS) oder Plazebo in einer für die Darmbesiedlung ausreichend großen Menge. Gleichzeitig wurde die Kuhmilch aus den Speiseplänen eliminiert. Nach vier Wochen hatten die
Kinder mit einem IgE-assoziierten atopischen Ekzem unter der Einnahme von LGG höhere Spiegel an C-reaktivem Protein (CRP) als die Plazebogruppe. Parallel dazu stiegen die IL-6-Spiegel nach der LGG-Behandlung an, was bei der Einnahme der Probiotika-Mischung oder von Plazebo nicht der Fall war. Bei den Kindern mit einer IgE-vermittelten Kuhmilchallergie waren die Spiegel des löslichen E-Selectins unter der Gabe der Probiotika höher als unter Plazebo.

Die leicht erhöhten Spiegel von IL-6 und CRP unter LGG weisen auf eine subklinische Entzündungsreaktion im Darm hin, induziert insbesondere durch
Wurde die Zahl der Atemwegsinfektionen herausgerechnet, blieb ein statistisch signifikanter Zusammenhang zwischen externer Tagesbetreuung und erhöhtem Allergierisiko bestehen.

Die Studie stellt einen Faktor der Hygienehypothese in Frage, kann aber nur wenig zur Analyse der Kofaktoren der Allergieentwicklung beitragen. So kamen zwar weniger Kinder in Tagesbetreuung aus ländlichen Gebieten und weniger hatten ein Haustier - beides nach der Hygienehypothese mögliche protektive Faktoren -, sie hatten aber nicht weniger Geschwister oder häufiger rauchende Eltern und sie waren auch nicht kürzer gestillt worden als die übrigen Kinder.

Fazit: Die Kinderbetreuung in Tagesstätten scheint nicht nur die Zahl der Infektionen, sondern auch das Risiko allergieverdächtiger Symptome zu erhöhen. Schwachpunkt ist, dass keine exakte Allergiediagnostik erfolgte.

Hagerhed-Engman L et al. Day-care attendance and increased risk for respiratory and allergic symptoms in preschool age. Allergy 2006; 61: 447-53

das Monozyten-Makrophagen-System. Dieses Ergebnis lässt vermuten, dass die Stimulation des angeborenen Immunsystems zumindest teilweise zum klinischen Effekt von LGG beitragen könnte. Dass dieser immunstimulierende Effekt aber nur bei Kindern mit Neurodermitis, nicht aber bei Kindern mit einer Kuhmilchallergie gefunden wurde, weist auf eine Beteiligung individueller Faktoren hin, die die immunologischen Effekte der probiotischen Bakterien modulieren.

Fazit: Probiotika stimulieren bei Kindern die intestinale Immunreaktion. Die dadurch induzierte niedriggradige Entzündung könnte zur Abschwächung der allergischen Reaktion beitragen. $b k$

Viljanen $\mathbf{M}$ et al. Induction of inflammation as a possible mechanism of probiotic effect in atopic eczema-dermatitis syndrome. J Allergy Clin Immunol 2005; 115: $1254-9$ 